\title{
La nouvelle francophone, marginalités, identités, singularités, éd. Thierry Oswald
}

\section{Elena Pessini}

\section{(2) OpenEdition}

1 Journals

\section{Édition électronique}

URL : http://journals.openedition.org/studifrancesi/5451

DOI : 10.4000/studifrancesi.5451

ISSN : 2421-5856

Éditeur

Rosenberg \& Sellier

\section{Édition imprimée}

Date de publication : 1 décembre 2016

Pagination : $570-571$

ISSN : 0039-2944

\section{Référence électronique}

Elena Pessini, « La nouvelle francophone, marginalités, identités, singularités, éd. Thierry Oswald », Studi Francesi [En ligne], 180 (LX | III) | 2016, mis en ligne le 01 janvier 2017, consulté le 18 septembre 2020 URL : http://journals.openedition.org/studifrancesi/5451; DOI : https://doi.org/10.4000/studifrancesi. 5451

Ce document a été généré automatiquement le 18 septembre 2020.

\section{(c)}

Studi Francesi è distribuita con Licenza Creative Commons Attribuzione - Non commerciale - Non opere derivate 4.0 Internazionale. 


\title{
La nouvelle francophone, marginalités, identités, singularités, éd. Thierry Oswald
}

\author{
Elena Pessini
}

\section{RÉFÉRENCE}

La nouvelle francophone, marginalités, identités, singularités. Actes du colloque de Limoges 13 mai 2014, textes réunis et présentés par Thierry osWALD, avec la collaboration de Thomas BAUER, Paris, Eurédit, 2015, pp. 194.

1 L'objectif que se sont fixé les organisateurs du colloque de Limoges tient du véritable défi et ils en sont bien conscients. Ils affirment d'ailleurs, dès l'Avant-propos qu'«Essayer d'évaluer l'importance et la fonction de la nouvelle dans les pays francophones, en proposer un petit historique, identifier des courants, des pionniers, des maîtres du genre, etc... peut paraître une gageure» (p. viII). Même si, comme on pouvait s'en douter, cet ouvrage est loin d'embrasser l'ensemble de la production francophone, il pose une série de problématiques qui concernent aussi bien les littératures en langue française que le genre de la nouvelle. L'Avant-propos, intitulé «Nouvelles en archipel», signé par Thierry OzWALD, précise les intentions de la recherche mais propose aussi au lecteur un parcours chronologique et géographique sur la naissance de la nouvelle francophone ainsi qu'une analyse des rapports entre le genre de la nouvelle et le genre romanesque. Il en ressort qu'il serait sans doute par trop osé de proposer une spécificité de la nouvelle francophone, ce dernier adjectif recouvre une aire géographique et culturelle beaucoup trop vaste pour qu'une seule étiquette puisse être suffisante. L'auteur souligne d'ailleurs d'emblée qu'il faut absolument tenir compte de l'existence de deux grands sous ensembles dans la francophonie. Un "premier cas», "celui des pays anciennement colonisés par la France - le Québec - ou bien soumis à l'influence culturelle directe de la France au fil 
des vicissitudes de l'histoire mais singularisés en dépit de leur inscription dans un même devenir historique depuis le Moyen Âge - la Belgique, la Suisse» (p. x) et un «second cas», «celui des pays récemment colonisés ou soumis à l'influence de la France: l'Afrique noire (le Mali, le Niger, le Sénégal, la Côte d'Ivoire, le Togo, le Cameroun, le Gabon, le Congo, etc...), le Maghreb; le Liban; l'Égypte, Djibouti, Haïti; Madagascar, la Réunion, l'île Maurice, les Antilles françaises, le Viêt Nam» (p. xI). Avant de se poser la question de l'existence d'une spécificité de la nouvelle francophone dans ces deux grandes zones, Thierry Ozwald s'interroge sur les problématiques du mimétisme et de l'imitation qui, comme chacun sait, constituent la première étape de ces littératures qui ne se forgent que dans un second temps sur le refus, la contestation, le détachement d'un modèle considéré au départ comme incontournable. Le paragraphe intitulé «Modalités de la nouvelle francophone» aborde, toujours au sein de cette réflexion introductive, le cœur du sujet et avance l'approfondissement le plus original - même s'il peut prêter à discussion, voire à contestation. La nouvelle francophone présenterait trois traits caractéristiques particuliers qui justifieraient qu'elle ait une identité distincte par rapport à la nouvelle franco-française: elle témoigne d'une identité collective, elle présente le leitmotiv de la femme sacrifiée et, en dernier lieu, la dialectique du rapport France/région francophone semble charpenter les textes. Pour suivre ces pistes de recherche, le classement des interventions au sein du colloque n'advient ni selon un ordre chronologique ni selon un ordre géographique, mais selon trois axes principaux qui constituent les trois parties de l'ouvrage: «La nouvelle, une poétique de l'indicible», «Étrangetés et dissidence», «Nouvelles et témoignages: réalités sociologiques». C'est sur la littérature québécoise que se penche l'intervention de claude FILTEAU, Savoir mourir, une affaire de culture: étude de la nouvelle de Gabrielle Roy, «Les satellites», qui inaugure la première section. L'écrivaine québécoise a choisi une forme brève pour mettre en scène les conflits qui naissent du choc de la rencontre entre la société innuit et le contexte canadien occidental. En particulier, les rituels liés au moment de la maladie et du trépas diffèrent profondément d'un groupe social à l'autre et les femmes sont davantage victimes des violences qui sont le fruit de ces situations conflictuelles. virginie BRINKER, elle, s'interroge sur les textes qui ont essayé de mettre en scène le génocide des Tutsis, à la lumière de la théorie du «désart» d'Adorno: Textes pour le Rwanda d'Abdourahman Waberi, Rêves sous le linceul de Jean-Luc Raharimanana, L'Iguifou et ce que murmurent les collines. Nouvelles rwandaises de Scholastique Mukasonga. Les écrivains, au-delà des choix individuels pour témoigner d'une tragédie, ne se posent pas seulement la question de savoir s'il est légitime ou pas de raconter l'indicible (question qui hante les écrivains chaque fois que, au cours de l'Histoire, les hommes repoussent un peu plus loin les limites de ce qu'on pensait qu'ils pouvaient accomplir comme violences sur leurs semblables) mais tous trois semblent donner à la littérature un rôle de premier plan dans la tentative de dépasser le simple constat des horreurs perpétrées. Tout au long de son analyse, Virginie Brinker conduit le lecteur vers une conclusion qui fournit un apport précieux aux débats soulevés lors du colloque: «C'est précisément en cela que la nouvelle nous semble l'un des genres les plus propices pour écrire le génocide, c'est-à-dire à la fois le chaos, certes, mais en même temps prouver qu'écrire n'est pas un acte vain, quoiqu'il le paraisse de prime abord. Écrire serait un geste fondamental, celui de réinscrire lecteurs et scripteurs dans une humanité qui, à défaut de retrouver l'harmonie d'un monde primitif ou de bien délimiter le bien et le mal, semble être la condition préalable d'une éventuelle renaissance» (p.30). Le rapport privilégié entre représentation de la violence et forme de la nouvelle est 
également abordé dans l'article suivant, signé Lidia ANoLL, intitulé Manifestations de la violence dans quelques récits d'Anne Hébert. Cette clé de lecture a déjà été appliquée à l'œuvre hébertienne par beaucoup de critiques mais ici, c'est une production considérée mineure qui est examinée: L'Ange de Dominique, La Robe de corail, Le Printemps de Catherine et La Mort de Stella, qui appartiennent au recueil Le Torrent, présentent à la fois des situations de violence entre les personnages et au sein de la nature, entre les éléments. Cette représentation permet de dénoncer l'inacceptable du monde; l'écrivaine exprime son refus de la résignation et fait de l'écriture un acte de révolte.

2 La deuxième partie de l'ouvrage souligne dans quelle mesure la forme brève de la nouvelle est le lieu littéraire privilégié où peut s'exprimer la voix des minorités, des dissidences aussi. Il peut s'agir d'une esthétique particulière; dans cette optique deux cas sont analysés au sein de la première et de la seconde intervention. Dans La nouvelle fantastique comme expression d'identité littéraire belge: l'œuvre de Bernard Quiriny, Alexandre KOMANDERA, après un bref excursus historico-littéraire visant à saisir le moment de la naissance et les modalités de l'affirmation d'un genre tout à fait spécifique de la littérature belge, la nouvelle fantastique, se concentre sur le cas particulier de Quiriny pour montrer «à quel point [il] suit les modèles et les règles du fantastique traditionnel et à quel point il les transgresse aussi pour élaborer sa propre esthétique» (p. 59). Pedro MENDEZ montre, lui aussi, à travers un approfondissement sur l'écrivain canadien Georges Boucher de Boucherville comment l'entrée dans les lettres canadiennes des thématiques et des formes liées au fantastique et la naissance du genre de la nouvelle sont étroitement liées. Les dissidences se font aussi entendre à travers l'ironie et l'humour. Sanae EL OUARDIRHI examine l'œuvre de l'écrivain marocain Fouad Laroui et en particulier son recueil qui a remporté en 2013 le prix Goncourt de la nouvelle: L'Étrange affaire du pantalon de Dassoukine. Le critique dans Fragmentation, absurde et dérision dans les nouvelles de Fouad Laroui, se fixe comme objectif de montrer comment le genre de la nouvelle permet à l'écrivain, mieux que toute autre forme narrative, de tourner en ridicule toutes les formes de la bêtise humaine, en particulier celle qui émerge dans les rapports entre colonisateur et colonisé mais aussi dans ses formes quotidiennes de la vie de couple ou des rapports familiaux. Laroui confie d'ailleurs luimême: «J'écris pour dénoncer des situations qui me choquent. Pour dénoncer la bêtise sous toutes ses formes» (p. 91). Qu'en est-il de la nouvelle aux Antilles? C'est la question que se pose Thierry ozwaLd dans son article Suzanne Dracius, Maryse Condé: «Pays mêlé», «Rue Monte au Ciel»: nouvelles et féminisme antillais. Cet approfondissement consacré à la Caraïbe, basé sur la comparaison et la mise en parallèle de deux écritures féminines, l'une guadeloupéenne et l'autre martiniquaise, permet de poser une série de différences entre les deux littératures qui sont souvent sinon confondues, au moins assimilées. En Martinique en particulier, la nouvelle semble avoir du mal à se frayer un chemin puisqu'elle doit se mesurer au genre dit noble du roman qui compte des noms aussi importants que Glissant, Chamoiseau et Confiant. (Ozwald ne manque d'ailleurs pas d'égratigner au passage ces écrivains dont il fait, nous semble-t-il parfois un portrait sans doute un peu forcé). Les nouvelles écrites par des femmes des deux îles ont cependant beaucoup de points communs, surtout dans leur effort de: «tenter de cerner, de définir, de donner corps à l'identité de la femme antillaise, ce qui revient à la libérer, à la faire accéder à sa liberté» (p. 112).

3 La dernière section analyse des œuvres qui se posent comme objectif de coller au réel, de photographier des réalités sociales en souffrance ou porteuses de malaises. C'est le 
cas dans le premier article, Portrait de l'Afrique en ballon rond, qui réfléchit sur un recueil publié en 2010, écrit en collaboration par un groupe d'auteurs en même temps que se déroulait la première coupe du monde de football sur le continent africain, intitulé Enfants de la balle. Nouvelles d'Afrique, nouvelles de foot. Préfacé par Abdourahman A. Waberi, l'ouvrage présente une série de situations qui ont toutes partie liée avec le monde du football mais en particulier avec le football africain et toutes les difficultés que peuvent connaitre de jeunes joueurs qui doivent quitter leur pays pour suivre un rêve qui, souvent, se transforme en cauchemar. La publication de ce recueil permet à Thomas BAUER d'affirmer que non seulement il existe une «littérature sportive» mais que «la nouvelle sportive africaine» a vu le jour. Kveta KUNESOvA, dans l'article qui suit, centre son attention sur le cas de Marco Micone, écrivain québécois, né en Italie et émigré avec sa famille en terre canadienne. La nouvelle hybride: Marco Micone montre comment la forme brève est particulièrement apte à décrire les souffrances de l'exil, les difficultés de l'arrachement à la terre d'origine mais aussi les tentatives de tracer les contours d'une nouvelle identité, multiple et hybride. La dernière contribution, Société morte, vive lamentation, signée Jean ARROUYE conduit une analyse très détaillée du recueil Propos changeants sur l'amour de la nouvelliste et romancière tunisienne Azza Filali où il apparait clairement que l'instrument narratif est mis au service de la dénonciation des travers de la société bourgeoise tunisienne: l'hypocrisie et l'égoïsme.

4 Tout en ayant aucune prétention d'exhaustivité, ce recueil a le mérite d'indiquer un certain nombre de pistes de réflexion et de travail mais aussi d'apporter un éclairage sur des œuvres qui n'ont pas toujours suscité l'attention de la critique mais qui sont toutefois particulièrement dignes d'intérêt. 